\title{
Manipulation of laser-accelerated proton beam profiles by nanostructured and microstructured targets
}

\author{
L. Giuffrida, ${ }^{1 *}$ K. Svensson, ${ }^{2}$ J. Psikal, ${ }^{1,3}$ M. Dalui, ${ }^{2}$ H. Ekerfelt, ${ }^{2}$ I. Gallardo Gonzalez, ${ }^{2}$ \\ O. Lundh, ${ }^{2}$ A. Persson, ${ }^{2}$ P. Lutoslawski, ${ }^{1}$ V. Scuderi, ${ }^{1,4}$ J. Kaufman, ${ }^{1}$ T. Wiste, ${ }^{1}$ T. Lastovicka, ${ }^{1}$ \\ A. Picciotto, ${ }^{5}$ A. Bagolini, ${ }^{5}$ M. Crivellari, ${ }^{5}$ P. Bellutti ${ }^{5}$ G. Milluzzo, ${ }^{4}$ G. A. P. Cirrone, ${ }^{4}$ \\ J. Magnusson, ${ }^{6}$ A. Gonoskov, ${ }^{6}$ G. Korn, ${ }^{1}$ C-G. Wahlström, ${ }^{2}$ and D. Margarone ${ }^{1}$ \\ ${ }^{1}$ Institute of Physics ASCR, v.v.i (FZU), ELI-Beamlines project, 18221 Prague, Czech Republic \\ ${ }^{2}$ Department of Physics, Lund University, P.O. Box 118, S-221 00 Lund, Sweden \\ ${ }^{3}$ FNSPE, Czech Technical University in Prague, 11519 Prague, Czech Republic \\ ${ }^{4}$ Laboratory Nazionali del Sud, INFN, 95125 Catania, Italy \\ ${ }^{5}$ Micro-Nano Facility, Fondazione Bruno Kessler, 38123 Trento, Italy \\ ${ }^{6}$ Department of Physics, Chalmers University of Technology, 41296 Gothenburg, Sweden
}

(Received 9 December 2016; published 4 August 2017)

\begin{abstract}
Nanostructured and microstructured thin foils have been fabricated and used experimentally as targets to manipulate the spatial profile of proton bunches accelerated through the interaction with high intensity laser pulses $\left(6 \times 10^{19} \mathrm{~W} / \mathrm{cm}^{2}\right)$. Monolayers of polystyrene nanospheres were placed on the rear surfaces of thin plastic targets to improve the spatial homogeneity of the accelerated proton beams. Moreover, thin targets with grating structures of various configurations on their rear sides were used to modify the proton beam divergence. Experimental results are presented, discussed, and supported by 3D particle-in-cell numerical simulations.
\end{abstract}

DOI: 10.1103/PhysRevAccelBeams.20.081301

\section{INTRODUCTION}

Table-top laser systems, using the chirped-pulse amplification (CPA) technique, which were developed in the last decades, are able to reach ultrahigh intensities (above $10^{18} \mathrm{~W} / \mathrm{cm}^{2}$ ) through the generation of femtosecond laser pulses [1], thus offering new possibilities in the study of relativistic laser-matter interactions [2,3]. Among others, laser-driven ion acceleration is one of the most promising and intensively investigated research topics [4], where target normal sheath acceleration (TNSA) is the experimentally most investigated technique. TNSA is based on the relativistic interaction of a thin target and an intense laser pulse and can be used to accelerate protons to several tens of $\mathrm{MeV}$ [5-8]. The front surface of the target is ionized by the leading edge of the laser pulse, creating a plasma expanding from the target front. A significant part of the laser pulse energy is absorbed and heats the plasma electrons which subsequently propagate through the target. As these hot electrons exit the rear of the target, they set up very strong electrostatic sheath fields that ionize atoms and molecules present on the target rear surface. Positively charged

\footnotetext{
*orresponding author.

Lorenzo.Giuffrida@eli-beams.eu

Published by the American Physical Society under the terms of the Creative Commons Attribution 4.0 International license. Further distribution of this work must maintain attribution to the author(s) and the published article's title, journal citation, and DOI.
}

particles can subsequently be accelerated in these sheath fields in the target normal direction. It is the particles with the highest charge-to-mass ratio that are preferentially accelerated, which, under the present experimental conditions, are protons.

The energy of the accelerated protons depends on the strength of the sheath fields, which in turn scales with the hot electron temperature, and therefore the fraction of absorbed laser energy. It is thus possible to increase the proton energies by enhancing the absorption of laser energy at the front of the target. It has also been shown that the spatial divergence of the emitted protons is energy dependent, which is a consequence of the initial electron sheath shape on the rear of the target, such that the divergence increases with decreasing proton energy [9]. The beams of accelerated protons present unique characteristics, e.g. short bunch duration at the source ( ps) [10], low transverse emittance (few $10^{-3} \mathrm{~mm}$ mrad [11]), and ultrahigh dose rate $\left(\sim 10^{9} \mathrm{~Gy} / \mathrm{s}\right)$ [12], which make them potentially very attractive for multidisciplinary applications, such as radiation biology [13], hadron therapy [14], proton radiography [15,16], and fast ignition [17].

In previous works, it was demonstrated experimentally that nanospheres deposited on the target front surface can increase the temperature and the distribution of hot electrons generated during relativistic intensity laser-solid interaction and, as a consequence, enhance the energy and number of accelerated protons, as well as the spatial homogeneity of the beam [18]. It has also been shown that it is possible to enhance the maximum energy of 
laser-accelerated protons by introducing grating microstructures on the target front surface [19].

We have extended these previous investigations by a study where the spatial profile of the proton beam is manipulated, both in terms of divergence and spatial homogeneity, by introducing nanospheres or $\mu \mathrm{m}$-sized grating structures on the rear side of the target. The reported experimental results are supported by 3D particle-in-cell (PIC) numerical simulations.

\section{EXPERIMENTAL SETUP}

\section{A. Laser system and pulse focusing}

The multiterawatt laser in the Lund University is a CPA based Ti:sapphire system with a central wavelength of $800 \mathrm{~nm}$, capable of delivering approximately $2 \mathrm{~J}$ of energy per pulse (uncompressed) at $10 \mathrm{~Hz}$ repetition rate. The typical pulse duration after compression is $35 \mathrm{fs}$ with an amplified spontaneous emission pedestal having an intensity ratio of approximately $10^{-9}$, measured 120 ps prior to the main pulse. A deformable mirror is placed after the laser pulse compressor to correct wavefront aberrations up to, and including the $f / 3$ off-axis parabolic focusing mirror (OAP). The measured laser energy delivered into the target chamber was approximately $1 \mathrm{~J}$ per pulse during the present experimental study. The full width at half maximum of the laser focal spot was measured to be approximately $3.5 \mu \mathrm{m}$. After the OAP, but prior to the laser focus, a plasma mirror (PM), placed at Brewster's angle, was used to further improve the temporal contrast on the target. In the current experiment, the reflectivity of the plasma mirror was measured to be $(47 \pm 4) \%$ with an estimated temporal contrast ratio enhancement of 2 orders of magnitude [20].

After the PM, the p-polarized laser pulse was focused on target with an incidence angle of $45^{\circ}$ relative to the target normal. The laser peak intensity, in vacuum, was determined from the above-mentioned total pulse energy (after the PM), the measured pulse duration, and a recorded high dynamic-range image of the spatial intensity distribution in the focal plane. This results in a peak intensity of about $6 \times 10^{19} \mathrm{~W} / \mathrm{cm}^{2}$, neglecting any changes to the laser spot size introduced by the PM. A sketch of the setup is shown in Fig. 1(a).

\section{B. Targets}

Targets used during this experimental campaign are sketched in Figs. 1(b) and 1(c). Plastic (Mylar ${ }^{\mathrm{TM}}$ ) foils of $500 \mathrm{~nm}$ thickness, covered with monolayers of polystyrene nanospheres of $400 \mathrm{~nm}$ diameter, were produced using the technique described in [21] at the ELI Beamlines target laboratory. Flat plastic foils of $900 \mathrm{~nm}$ thickness, i.e. the same overall thickness as of the nanosphere-covered targets, were used for comparison. Free-standing silicon nitride (SiN) membranes of $700 \mathrm{~nm}$ thickness, developed and fabricated at Fondazione Bruno Kessler, were also used in the experiment. The SiN membranes were deposited by a plasma enhanced chemical vapor deposition reactor and

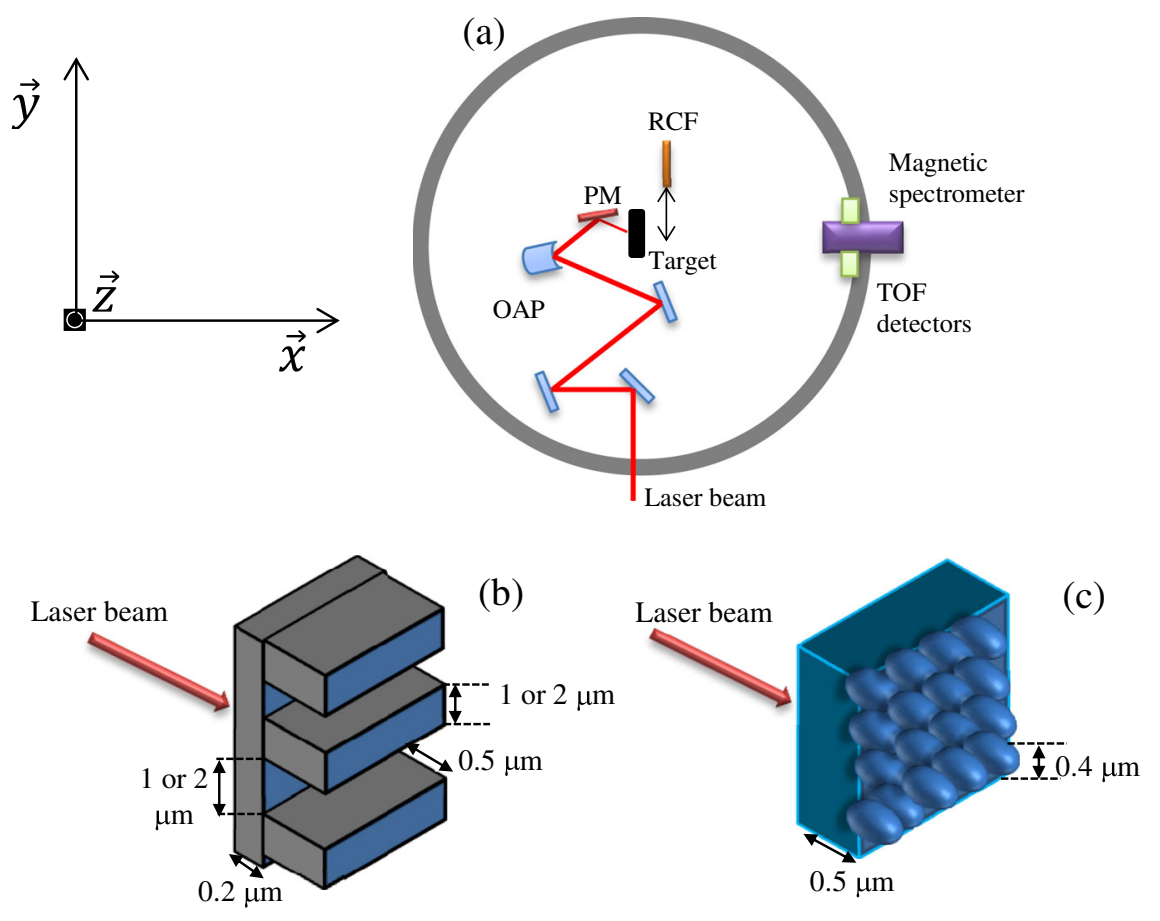

FIG. 1. Top view of the experimental setup (a), sketch showing the plastic target covered with nanospheres (b), and side view of the grating targets (c) used in the experiment. Here $\vec{x}$ is the axis normal to the target surface, $\vec{y}$ is the direction perpendicular to the orientation of grating ridges and $\vec{z}$ is the direction parallel to the orientation of horizontal grating ridges. 
defined by a chemical etching of the silicon wafer on their rear. Various grating structures, with different periodicities, were shaped on the SiN membranes. The ridges had widths of $(0.5 \pm 0.1) \mu \mathrm{m}$, while the grooves between them were $(0.5 \pm 0.1) \mu \mathrm{m}$ deep and $(1 \pm 0.1) \mu \mathrm{m}$ or $(2 \pm 0.2) \mu \mathrm{m}$ wide.

\section{Proton diagnostics}

Various detectors were employed to characterize the beams of laser-accelerated protons. A hyperpure $4.4 \times 4.5 \mathrm{~mm}^{2}$, $500 \mu \mathrm{m}$ thick, single crystal diamond detector (SCDD) was placed behind the target at a distance of $56 \mathrm{~cm}$ and an angle of $9^{\circ}$ with respect to the target normal. This detector was used in time-of-flight (TOF) configuration, which allows the energy spectrum of the accelerated proton beam to be indirectly determined. A picture of the used detector and its electric scheme are reported in Figs. 2(a) and 2(b), respectively. A magnetic spectrometer placed behind the target in the target normal direction was also used to retrieve proton energy spectra [a sketch is reported in Fig. 2(c)]. This spectrometer consists of a $1 \mathrm{~mm}$ entrance slit in front of a $5 \mathrm{~cm}$ permanent dipole magnet with $0.83 \mathrm{~T}$ mean field strength, deflecting protons according to their energies. Protons are then stopped in a plastic scintillator (Saint Gobain, BC-408 [22]) and the resulting fluorescence is imaged by a 16-bit electron multiplying charged coupled device camera. The scintillator is covered with a $6 \mu \mathrm{m}$ thick aluminum foil, which shields it from scattered laser light and optical radiation from the plasma. This foil also stops any low-energy ions of higher nuclear charge, such as carbon, oxygen, or silicon, due to its much higher stopping power for such ions compared to protons. Thanks to this foil, in combination with the energy, mass, and charge dependent dispersion in the magnetic field, as well as the nuclear charge dependent scintillator response, which is much lower for higher nuclear charge ions compared to protons, the observed signal becomes dominated by the contribution from accelerated protons only in the regime investigated in the present study. Finally, radiochromic film (RCF) stacks composed of a few layers of HD-V2 RCF type were used to characterize divergence and spatial uniformity of the accelerated proton beams. The RCF stacks were placed $6 \mathrm{~cm}$ behind the target in the target normal direction. The RCF stacks were covered by $2.4 \mu \mathrm{m}$ $\mathrm{Al}$ filter, in order to cut the protons with less than $275 \mathrm{keV}$ and the visible/UV radiation coming out from the plasma after the laser-target interaction. The positions of the detectors were such that it was not possible to obtain simultaneous information on the spatial profiles and on the energy distribution of the accelerated protons.

\section{EXPERIMENTAL RESULTS}

Plastic (Mylar or polyethylene terephthalate) foils of $900 \mathrm{~nm}$ thickness (PET) were used as reference targets in terms of accelerated proton beam features in the standard TNSA regime [5-8]. Results obtained with such targets were compared with the ones of proton beams generated from Mylar foils covered with nanospheres on the substrate
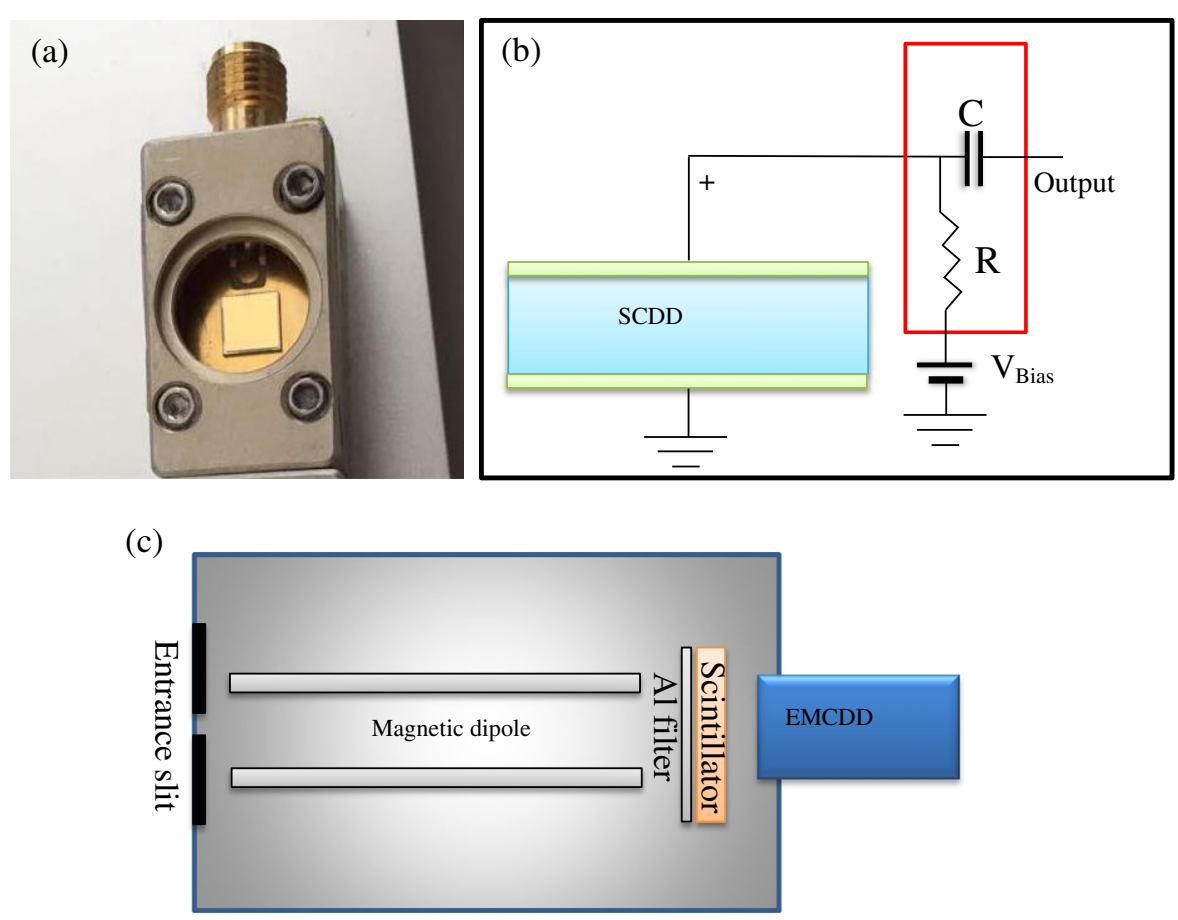

FIG. 2. Photo (a) and electrical scheme (b) of the SCDD detector, and sketch of the magnetic spectrometer (c) used during the experiment. 
rear side $\left(\mathrm{NS}_{\text {rear }}\right)$. Moreover, targets made of SiN membrane with a thickness of $200 \mathrm{~nm}(\mathrm{SiN})$ were used as reference, and targets with gratings on the rear side with a step of $1 \mu \mathrm{m}\left(\mathrm{SiN}_{1 \mu \mathrm{m}}\right)$ and $2 \mu \mathrm{m}\left(\mathrm{SiN}_{2 \mu \mathrm{m}}\right)$ were also used.

Figure 3 reports energy distributions obtained with the magnetic spectrometer [(a) and (c)] and TOF ion current distributions [(b) and (d)] measured by the SSCD detector for the different types of used targets. TOF measurements were useful during the experiment to control in real time the maximum energy (by identifying the beginning of the signal) and the proton beam flux. However they were placed at a larger angle compared to the magnetic spectrometer.

Figure 3(b) shows that the maximum proton energy was about 3.0 and $2.5 \mathrm{MeV}$ for PET and $\mathrm{NS}_{\text {rear }}$ targets, respectively. Such result is in agreement with magnetic spectrometer measurements, where the maximum proton energy was about 3.6 and $3.0 \mathrm{MeV}$ for PET and $\mathrm{NS}_{\text {rear }}$ targets, respectively. It is important to stress that such difference is attributed to the different detection angles of the two diagnostic systems $\left(0^{\circ}\right.$ for the spectrometer and $9^{\circ}$ for the SSCD detector). Figure 3(b) shows the maximum proton energy in the case of $\mathrm{SiN}$ and $\mathrm{SiN}_{2} \mu \mathrm{m}$ targets, which was about 3.0 and $2.3 \mathrm{MeV}$, respectively. Also in this case the TOF results are in agreement with the ones obtained with the magnetic spectrometer.
Figure 4 shows raw images of proton beam imprints on the RCF for two of the used target types: (a) PET and (b) $\mathrm{NS}_{\text {rear }}$, in both cases obtained accumulating two consecutive shots. Proton beam imprints for PET show a divergence in FWHM, of $\pm 5^{\circ}$. With $\mathrm{NS}_{\text {rear }}$ the situation is very different, and the divergence increases drastically up to $\pm 10^{\circ}$. Furthermore, proton beams emerging from these targets are spatially more homogeneous than beams generated with PET. The degree of inhomogeneity of proton beams emerging from PET is about $16 \%$, but only $8 \%$ in the case of $\mathrm{NS}_{\text {rear }}$. Such parameter was determined reconstructing a histogram of the beam dose profile. Then the standard deviation was calculated and divided by the mean which provided percentage value that describes the degree of homogeneity. The fact that the calculation is performed in dose domain is crucial as the intensity profile does not correspond to reality due to nonlinear response of the films. A comparison of the RCF imprints of protons emerging from the different targets ( $\mathrm{SiN}$, and $\mathrm{SiN}_{1 \mu \mathrm{m}}$ and $\mathrm{SiN}_{2} \mu \mathrm{m}$ ) is shown in Fig. 5. The proton beam imprint for $\mathrm{SiN}$ [Fig. 5(a)], obtained accumulating two consecutive shots, shows a divergence of about $\pm 4^{\circ}$. The shape of the proton imprint is very different when grating structures are present on the rear of the target [Figs. 5(b) and 5(c)], where in both cases RCFs were obtained only after one shot. The first important feature is that, in the direction parallel to the
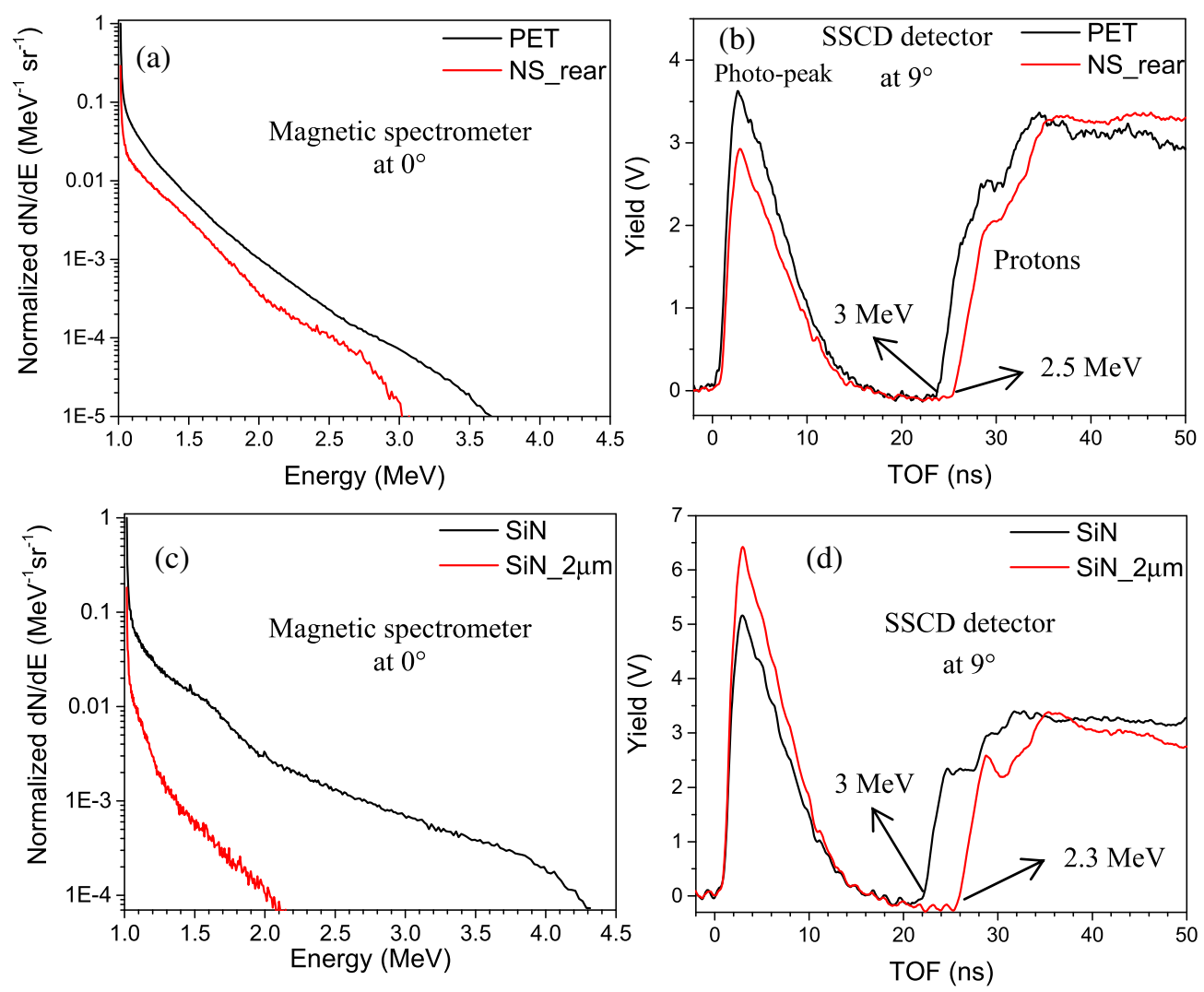

FIG. 3. Proton energy distributions measured by the magnetic spectrometer for PET and $\mathrm{NS}_{\text {rear }}$ (a) and for $\mathrm{SiN}_{\text {and }} \mathrm{SiN} \mathrm{N}_{2} \mu \mathrm{m}$ (c); the corresponding TOF spectra from the SSCD detector are shown in (b) and (d). 

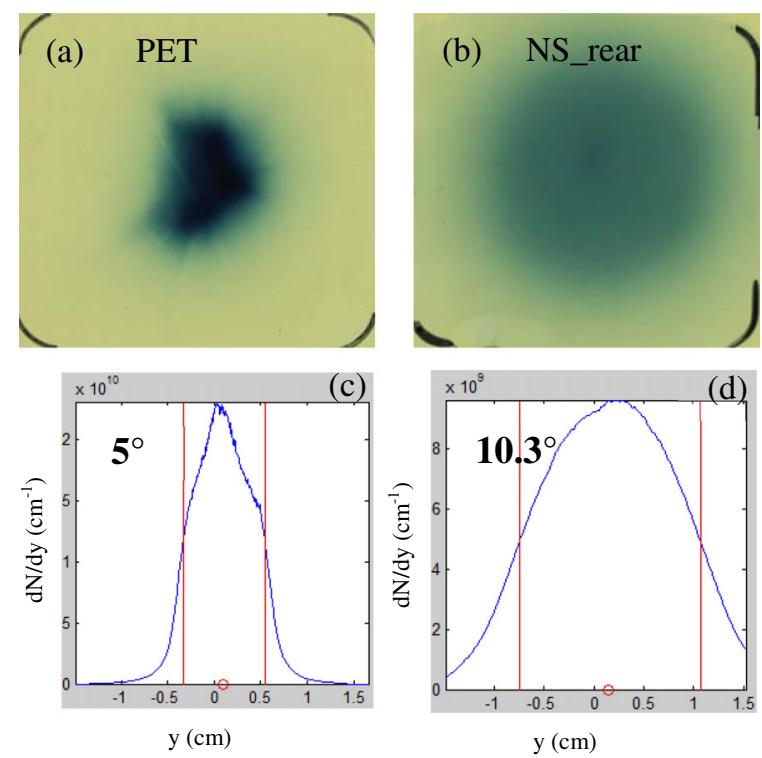

FIG. 4. Examples of radiochromic films (obtained accumulating 2 shots) showing spatial profiles of proton beams generated from PET target (a) and from $\mathrm{NS}_{\text {rear }}$ (b). (c) and (d) represent the lateral profile of the proton beam respectively for PET and $\mathrm{NS}_{\text {rear }}$ targets.

grating orientation, the divergence of the beam is reduced being about $\pm 4^{\circ}$ for $\mathrm{SiN}$ and ranging between $\pm 1^{\circ}$ and $\pm 2^{\circ}$ for $\mathrm{SiN}_{1 \mu \mathrm{m}}$ and $\mathrm{SiN}_{2 \mu \mathrm{m}}$ (statistical fluctuations in such a range are measured in different shots). The second very pronounced feature is observed in the direction perpendicular to the grating ridges, where the proton beam imprints are strongly stretched.

\section{SIMULATIONS}

In order to better understand the physics behind the experimental results, different sets of PIC simulations (2D and 3D) were performed with the PIC codes EPOCH [23] and PICADOR [24]. The ECLIPSE cluster built within the ELI-Beamlines project was used for the 3D simulations using the EPOCH code. Computing and storage facilities provided by the CERIT-SC Center were used for 2D simulations.

In the 3D simulations, which can fully describe the interaction geometry, we assumed a linearly p-polarized laser pulse with a Gaussian temporal profile of 30 fs (FWHM) and a Gaussian spatial profile of the beam with focal spot size of $4 \mu \mathrm{m}$ (FWHM). The peak intensity of the pulse was set to $2 \times 10^{19} \mathrm{~W} / \mathrm{cm}^{2}$, the laser beam was incident on target with a $45^{\circ}$ angle with respect to the target normal. The target is composed of a $\mathrm{C}^{6+} \mathrm{H}_{2}{ }^{+}$plasma with density of $40 n_{\mathrm{ec}}$, where $\mathrm{n}_{\mathrm{ec}}$ is the electron critical density. The initial target density profile was steplike in all cases. In the simulations, we assumed flat foils with a thickness of $700 \mathrm{~nm}$ (the same shape of the target as SiN target in the experiment) and grating target with the parameters shown in Fig. 1(b), i.e. with grooves of $0.5 \mu \mathrm{m}$ in depth and $2 \mu \mathrm{m}$ in width (the same shape of the target as SiN_2 $\mu \mathrm{m}$ target in the experiment). The sizes of cubic cells in 3D were equal to $16^{3} \mathrm{~nm}^{3}$ and the number of macroparticles per cell was set to 40 electrons, 10 protons, and $5 \mathrm{C}^{6+}$ ions. The simulation box has sizes of $20 \times 28 \times 16 \mu \mathrm{m}$.

The simulation results for $\mathrm{SiN}$ and $\mathrm{SiN}_{2} \mu \mathrm{m}$ presented in Fig. 6 show a good agreement with the experimental results. The simulations show a regular shape for the angular distribution of the accelerated protons from $\mathrm{SiN}$
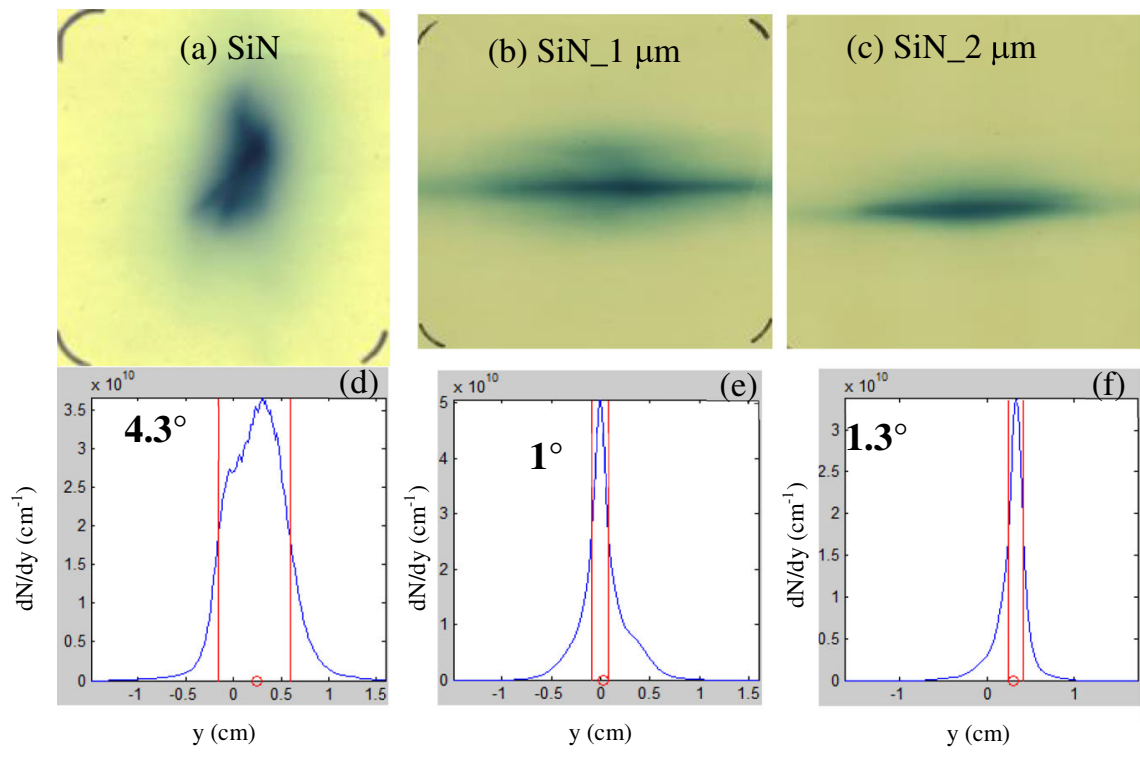

FIG. 5. Examples of radiochromic films showing the spatial profiles of proton beams emerging from SiN (obtained accumulating 2 shots) (a), from a $\operatorname{SiN}_{1 \mu \mathrm{m}}$ (1 shot) (b) and from a $\mathrm{SiN}_{2 \mu \mathrm{m}}$ (1 shot) (c) targets. (d),(e),(f) show the lateral profile of the proton beam for the different targets in (a)-(c). 
(a)
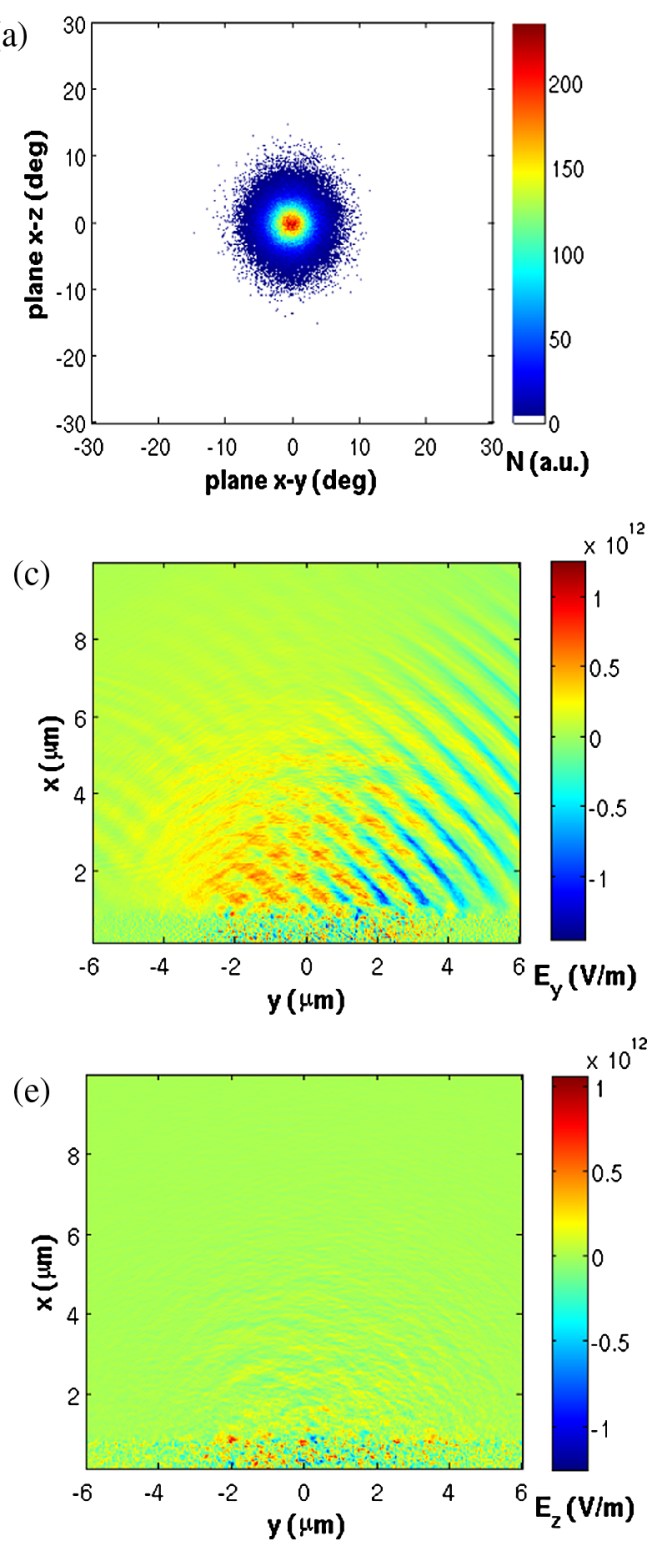

(b)
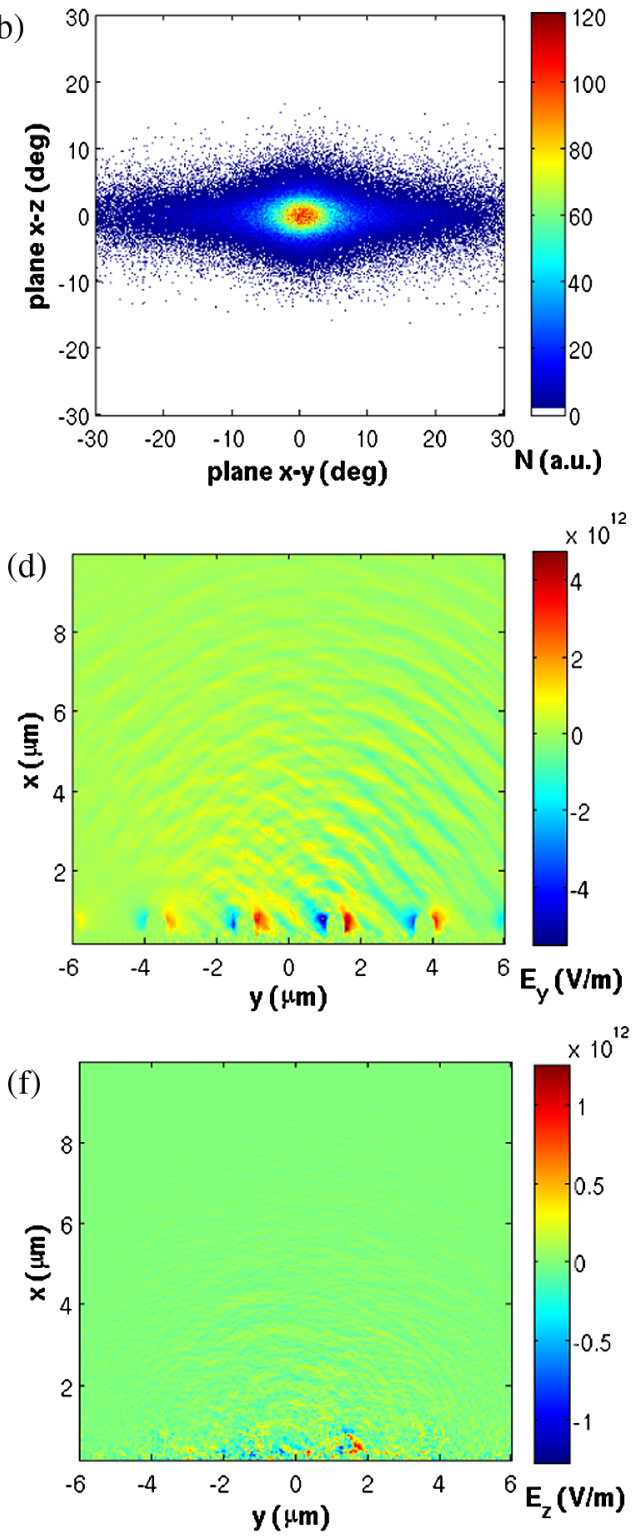

FIG. 6. Angular distribution of accelerated protons from 3D PIC simulations for $\mathrm{SiN}$ (a) and $\mathrm{SiN}_{2} \mu \mathrm{m}$ (b). (a), (b) Horizontal axis: deflection angle from the target normal direction ( $x$ axis) in the plane $x-y$; vertical axis: deflection angle from the target normal direction ( $x$ axis) in the plane $x-z$; colorbar: number of numerical macroparticles per spatial angle in simulations. Electric field perpendicular to the grating orientation for $\mathrm{SiN}$ (c) and $\mathrm{SiN}_{2 \mu \mathrm{m}}$ (d) and electric field parallel to the grating orientation for $\mathrm{SiN}$ (e) and $\mathrm{SiN}_{2 \mu \mathrm{m}}$ (f). The target front (laser-irradiated) surface is at $x=0$, slightly outside the area shown in (c)-(f) (the laser comes from the bottom).

[see Fig. 6(a)] and a large angular spread perpendicular to the grating orientation (in the $y$ axis direction) for the structured target $\left(\mathrm{SiN}_{2} \mu \mathrm{m}\right)$ [see Fig. 6(b)]. These results are better explained in Figs. 6(c) and 6(d), where the electric field in the $y$ axis direction is reported for the two targets. In Fig. 6(d) a transverse component of the electric field is present, which is mainly responsible for stretching the proton beam in the transverse direction. Figures 6(e) and 6(f) show the electric field in the $z$ axis direction for $\mathrm{SiN}$ and $\mathrm{SiN}_{2 \mu \mathrm{m}}$ targets, respectively. They present very similar features, thus they cannot explain the observed difference in proton beam divergence in the $z$ direction, i.e. parallel to the grating grooves. However, it is clear from Figs. 6(a) and 6(b) that the density of the proton beam in the case of the SiN target is several times larger than in the case of the $\mathrm{SiN}_{2 \mu \mathrm{m}}$ [the number of accelerated protons per unit solid angle in (a) is twice larger than in (b) in the middle of the beam]. Thus, a possible explanation for the different divergence measured experimentally for the flat and grating targets could be that the higher proton density at the source (in proximity of the target) for SiN can result in a higher divergence of the accelerated proton beam due to a larger 
Coulomb repulsion between particles during the beam expansion. Similar results were observed for the geometric emittance calculated from 3D simulations, which was estimated to be about $0.45 \mathrm{~mm}$ mrad in the $z$ direction for both targets in the case of high-energy protons (above $2 \mathrm{MeV}$ ).

These results were also independently confirmed by 2D simulations using the codes EPOCH [23] and PICADOR [24] with similar parameters of the interaction in both 2D simulations. The results from 2D simulations qualitatively agree with 3D calculations in the influence of the presence of grating structures on the rear surface of the target in terms of corresponding proton angular distribution.

\section{DISCUSSION AND CONCLUSIONS}

The possibility to manipulate the spatial profiles of proton beams generated by the interaction of an intense femtosecond laser pulse and advanced targets with surface nano- or microstructures on the rear side has been experimentally and numerically explored.

Nanospheres covering the rear surface of a flat plastic foil affect the final proton beam spatial profile in terms of beam divergence and homogeneity, such that it has a larger divergence and a more homogeneous spatial distribution compared to a proton beam emerging from a flat plastic foil of equivalent total thickness. This effect can be explained through a larger transverse emittance of the generated proton beam due to the microcurved surface, which smoothes down beam inhomogeneity compared to flat targets.

Grating structures of $\mu \mathrm{m}$ size covering the rear of silicon nitride targets were also used to modify the proton beam spatial distribution, reducing its divergence in the direction parallel to the grating orientation and stretching it in the perpendicular direction due to transverse electric fields generated inside the target grooves. This effect is completely new and according to our knowledge it was never investigated before. The behavior of these targets is completely different from that of targets described in [25], where metallic foils covered by sinusoidal microgrooves were irradiated with the aim of measuring the source size of the laser-accelerated protons for a given energy. In fact those targets were much thicker and the dimensions and size of the grooves were very different compared to the grating targets used in our investigations. Moreover, the most crucial difference lies in the laser pulse parameters. Reference [25] reports pulse duration of few hundreds of fs, whereas 35 fs pulses were used in our experiment. Since TNSA regime takes place during the laser-target interaction and the acceleration of ions is stopped shortly after the interaction, the front of the accelerated proton stream keeps the initial structure of the rear side of the target for ultrashort pulses during the whole acceleration time. In this case, the sheath is not homogeneous and the protons are accelerated both along the target normal direction and into the transverse direction as well. This phenomenon is illustrated by a strong transverse electric field generated due to rear-side structures [see Fig. 6(d)]. In the case of much longer laser pulses, e.g. in [25], the structures on the proton beam front are gradually smoothed and protons are mostly accelerated by a homogenized sheath along the target normal direction. Therefore, a modulated rear surface is here translated only into strip structures appearing in the proton beam spatial profile (as can be seen in Fig. 1 in [25] or in Fig. 2 in [26], but not in a larger proton beam divergence).

The present experimental results are confirmed by numerical simulations performed by using $2 \mathrm{D}$ and $3 \mathrm{D}$ PIC codes. A possible explanation of the observed reduction in proton divergence is Coulomb repulsion between charged particles during the proton beam expansion, which might be larger for the flat foil due to larger density of ions in the beam. However, this effect is not easily predictable by the PIC simulations because they show proton acceleration and propagation only up to a distance of a few $\mu \mathrm{m}$ from the target, i.e. where this effect is not yet fully developed.

Protons accelerated from targets with micro- and nanostructures on their rear side could be useful for applications in multidisciplinary research fields. In fact, the controlled divergence and the enhanced spatial homogeneity can be beneficial when using the generated proton beam for radiography [15] or cell irradiation [27,28], especially in the case of large objects to be investigated with a high temporal resolution, thus to be placed close to the source of nonrelativistic protons.

\section{ACKNOWLEDGMENTS}

This work has been supported by the project ELIExtreme Light Infrastructure-phase 2 (CZ.02.1.01/ 0.0/0.0/15_008/0000162) from European Regional Development Fund, the Ministry of Education, Youth and Sports of the Czech Republic (project No. LQ1606), the Czech Science Foundation (project No. 15-02964S), LASERLAB-EUROPE (Grant Agreement No. 284464, EC's Seventh Framework Programme), the Swedish Research Council and the Knut and Alice Wallenberg foundation, including its funding of the PLIONA project. Access to the CERIT-SC computing and storage facilities provided by the CERIT-SC Center, provided under the program "Projects of Large Research, Development, and Innovations Infrastructures" (CERIT Scientific Cloud LM2015085), is greatly appreciated.

[1] D. Strickland and G. Mourou, Compression of amplified chirped optical pulses, Opt. Commun. 56, 219 (1985).

[2] G. Morou, C. Labaune, M. Dunne, N. Naumova, and V. Tikhonchuk, Relativistic laser-matter interaction: from attosecond pulse generation to fast ignition, Plasma Phys. Control. Fusion 49, B667 (2007). 
[3] Y. I. Salamin, S. Hu, K. Hatsagortsyan, and C. Keitel, Relativistic high-power laser-matter interactions, Phys. Rep. 427, 41 (2006).

[4] V. Malka and P. Mora, Principles of laser-plasma accelerators, Les accélérateurs de particules laser-plasma, C.R. Phys. 10, 106 (2009).

[5] M. Passoni, L. Bertagna, and A. Zani, Target normal sheath acceleration: theory, comparison with experiments and future perspectives, New J. Phys. 12, 045012 (2010).

[6] S. C. Wilks, A. B. Langdon, T. E. Cowan, M. Roth, M. Singh, S. Hatchett, M. H. Key, D. Pennington, A. MacKinnon, and R.A. Snavely, Energetic proton generation in ultraintense laser-solid interactions, Phys. Plasmas 8, 542 (2001).

[7] S. P. Hatchett et al., Electron, photon, and ion beams from the relativistic interaction of Petawatt laser pulses with solid targets, Phys. Plasmas 7, 2076 (2000).

[8] F. Wagner et al., Maximum Proton Energy above $85 \mathrm{MeV}$ from the Relativistic Interaction of Laser Pulses with Micrometer Thick CH2 Targets, Phys. Rev. Lett. 116, 205002 (2016).

[9] D. C. Carroll et al., Active manipulation of the spatial energy distribution of laser-accelerated proton beams, Phys. Rev. E 76, 065401 (2007).

[10] B. Dromey et al., Picosecond metrology of laser-driven proton bursts, Nat. Commun. 7, 10642 (2016).

[11] T. E. Cowan et al., Ultralow Emittance, Multi-MeV Proton Beams from a Laser Virtual-Cathode Plasma Accelerator, Phys. Rev. Lett. 92, 204801 (2004).

[12] K. W. D. Ledingham, P. R. Bolton, N. Shikazono, and C.-M. C. Ma, Towards Laser Driven Hadron Cancer Radiotherapy: A Review of Progress, Appl. Sci. 4, 402 (2014).

[13] A. Yogo et al., Development of laser-driven quasi-monoenergetic proton beam line for radiobiology, Nucl. Instrum. Methods Phys. Res., Sect. A 653, 189 (2011).

[14] M. Durante and J. S. Loeffer, Charged particles in radiation oncology, Nat. Rev. Clin. Oncol. 7, 37 (2010).

[15] J. Mackinnon et al., Proton Radiography of a Laser-Driven Implosion, Phys. Rev. Lett. 97, 045001 (2006).

[16] M. Borghesi, A. Schiavi, D. H. Campbell, M. G. Haines, O. Willi, A. J. MacKinnon, L. A. Gizzi, M. Galimberti, R. J. Clarke, and H. Ruhl, Proton imaging: a diagnostic for inertial confinement fusion/fast ignitor studies, Plasma Phys. Control. Fusion 43, A267 (2001).

[17] M. Temporal, R. Ramis, J. J. Honrubia, and S. Atzeni, Fast ignition induced by shocks generated by laser-accelerated proton beams, Plasma Phys. Control. Fusion 51, 035010 (2009).

[18] D. Margarone et al., Laser-Driven Proton Acceleration Enhancement by Nanostructured Foils, Phys. Rev. Lett. 109, 234801 (2012); D. Margarone et al., Laser-driven high-energy proton beam with homogeneous spatial profile from a nanosphere target, Phys. Rev. ST Accel. Beams 18, 071304 (2015).

[19] T. Ceccotti et al., Evidence of Resonant Surface-Wave Excitation in the Relativistic Regime through Measurements of Proton Acceleration from Grating Targets, Phys. Rev. Lett. 111, 185001 (2013).

[20] G. Doumy, F. Quéré, O. Gobert, M. Perdrix, P. Martin, P. Audebert, J. C. Gauthier, J.-P. Geindre, and T. Wittmann, Complete characterization of a plasma mirror for the production of high-contrast ultraintense laser pulses, Phys. Rev. E 69, 026402 (2004).

[21] L. Stolcova, J. Proska, F.Novotny, M. Prochazka, and I. Richter, Periodic arrays of metal nanobowls as SERSactive substrates, in NANOCON 2011 (2011), vol. 1, pp. 737-741.

[22] Saint-Gobain website: http://www.crystals.saint-gobain .com/sites/imdf.crystals.com/files/documents/ organics-brochure.pdf.

[23] T. D. Arber et al., Contemporary particle-in-cell approach to laser-plasma modelling, Plasma Phys. Control. Fusion 57, 113001 (2015).

[24] S. Bastrakov, R. Donchenko, A. Gonoskov, E. Efimenko, A. Malyshev, I. Meyerov, and I. Surmin, Particle-in-cell plasma simulation on heterogeneous cluster systems, J. Comput. Sci. 3, 474 (2012).

[25] H. Ruhl, T. Cowan, and J. Fuchs, The generation of micro-fiducials in laser-accelerated proton flows, their imaging property of surface structures and application for the characterization of the flow, Phys. Plasmas 11, L17 (2004).

[26] T. E. Cowan et al., Ultralow Emittance, Multi-MeV Proton Beams from a Laser Virtual-Cathode Plasma Accelerator, Phys. Rev. Lett. 92, 204801 (2004).

[27] S. D. Kraft et al., Dose-dependent biological damage of tumour cells by laser-accelerated proton beams, New J. Phys. 12, 085003 (2010).

[28] D. Doria et al., Biological effectiveness on live cells of laser driven protons at dose rates exceeding $10^{9} \mathrm{~Gy} / \mathrm{s}$, AIP Adv. 2, 011209 (2012). 